\title{
A CONVENIENT MODEL CATEGORY FOR COMMUTATIVE RING SPECTRA
}

\author{
BROOKE SHIPLEY
}

\begin{abstract}
We develop a new system of model structures on the modules, algebras and commutative algebras over symmetric spectra. In addition to the same properties as the standard stable model structures defined in [HSS] and [MMSS], these model structures have better compatibility properties between commutative algebras and the underlying modules.
\end{abstract}

\section{INTRODUCTION}

In this paper we develop the $S$-model structure on symmetric spectra where $S$ is the sphere spectrum and the associated $R$-model structures on $R$-modules, associative $R$-algebras, and commutative $R$-algebras. The $S$-model structure was defined in [HSS, 5.3.6], but the axioms for a model category were not verified there. These model structures have all of the same properties as the standard stable model structures developed in [HSS] and [MMSS]. The advantage though of this system of model structures is the unique compatibility between the model structures on commutative ring spectra and on the underlying spectra.

This compatibility does not exist in any of the model structures already constructed on the various symmetric monoidal categories of spectra and their associated commutative monoids [EKMM, HSS, MMSS, Man]. This compatibility property has already been useful in [Sch01, 4.3] and [GS], and may have helped with some of the technical points in [Bas99, Section 9]. As discussed in Remark 2.9, analogues of the $S$-model structures here could also be constructed on orthogonal spectra and commutative orthogonal ring spectra as well.

The first special property of the $R$-model structures is that the associated cofibrations of commutative $R$-algebras are also $R$-cofibrations of the underlying $R$ modules; see Proposition 4.1. Also, the positive stable cofibrations of commutative $R$-algebras from the model categories developed in [MMSS, Man] are underlying $R$-cofibrations. This property has already been used in [Sch01]. (Note the $S$-cofibrations of $R$-modules from [Sch01] are the maps we refer to here as $R$-cofibrations.)

The second special property is an extension of the first and is the property required in [GS]. The cofibrations and fibrations in the $R$-model structure on commutative $R$-algebras forget to cofibrations and fibrations in a monoidal model structure on $R$-modules (namely the positive $R$-model structure, see Proposition 4.1 ). This property is not shared by the positive stable model structure on commutative $R$ algebras from [MMSS] or [Man]. Basically, this means that a cofibrant and fibrant replacement of a given commutative $R$-algebra is also cofibrant and fibrant as an

1991 Mathematics Subject Classification. 55P42, $55 \mathrm{U} 35$.

The author was partially supported by an NSF Grant and a Sloan Research Fellowship. 
underlying $R$-module. (There is a small technicality here which is dealt with in Section 4.) This is used in [GS] for constructing homotopy invariant mapping spectra in the category of $R$-modules while maintaining the extra commutative algebra structure on the source and target; see Corollary 4.6.

In Section 1 we develop a model structure on $G$-spaces which lies in between the usual naive and genuine equivariant model categories. In Section 2, we define model structures on symmetric sequences, symmetric spectra, module spectra and associative algebra spectra. We then consider commutative algebras in Section 3. In the final section, Section 4, we consider the special properties of the model structures developed in Sections 2 and 3.

We assume the reader is familiar with the standard model structures on symmetric spectra developed in [HSS] and [MMSS]. We also assume familiarity with the standard model category techniques and terminology; see [DS95] for an introduction and [Hov98] for a more thorough account. Specifically, to establish several of the model categories here we use the criterion for cofibrantly generated model categories from [Hov98, 2.1.19] which is based on [DHK]. Throughout the paper we work with categories built from simplicial sets which are thus locally presentable [Bor94, $5.2 .2 \mathrm{~b}, 5.3 .3,5.5 .9,5.7 .5]$. The smallness conditions in [Hov98, 2.1.19] then follow from [Bor94, 5.2.10] since each object is small.

Acknowledgments: Jeff Smith first defined the $S$-model structure on symmetric spectra. I'd like to thank him for encouraging me to develop this structure further. I had many useful conversations about this material with Mike Mandell and thank him for sharing his preprint [Man] with me. It was also an observation of his which led me to the current formulation of Corollary 4.5. I would also like to thank Stefan Schwede for several suggestions and questions about an earlier version of this paper.

\section{Equivariant SPACES}

There are two well-known model categories on the category of pointed $G$-equivariant simplicial sets (pointed $G$-spaces) for $G$ a finite group. In the naive version, weak equivalences and fibrations are determined on the underlying space. In the genuine equivariant model category we begin with below, the weak equivalences and fibrations are determined on the fixed point sets with respect to all subgroups $H$ in $G$.

Definition 1.1. A map $f$ of pointed $G$-spaces is an equivariant weak equivalence (equivariant fibration) if the induced map on the fixed points $f^{H}$ is a weak equivalence (fibration) of spaces for each subgroup $H$ of $G$.

Let $I^{\prime}=\left\{i_{k} \wedge(G / H)_{+}:(\partial \Delta[k] \times G / H)_{+} \rightarrow(\Delta[k] \times G / H)_{+}\right\}$for $k \geq 0$ and $J^{\prime}=\left\{j_{k}^{r} \wedge(G / H)_{+}:\left(\Lambda^{r}[k] \times G / H\right)_{+} \rightarrow(\Delta[k] \times G / H)_{+}\right\}$for $k>0,0 \leq r \leq k$, and $H$ varies over all subgroups of $G$ in both $I^{\prime}$ and $J^{\prime}$. Here $\Lambda^{r}[k]$ is the $r$ th horn of $\Delta[k]$, obtained by removing its $r$ th face. It is easy to check that the $J^{\prime}$-injectives are the equivariant fibrations and the $I^{\prime}$-injectives are the equivariant acyclic fibrations, i.e., the $J^{\prime}$-injectives which are also equivariant weak equivalences. One can also show that the $I^{\prime}$-cofibrations are the monomorphisms. After checking that each map in $J^{\prime}$ is an equivariant weak equivalence, [Hov98, 2.1.19] implies the following statement.

Proposition 1.2. The monomorphisms, equivariant weak equivalences and equivariant fibrations form a cofibrantly generated model structure on the category of pointed $G$-spaces. 
The model structure on pointed $G$-spaces which is of interest for the rest of this paper is a localization of this equivariant model structure which we refer to as the mixed model structure. The mixed cofibrations are again the monomorphisms, but the mixed weak equivalences are the underlying weak equivalences of spaces and the mixed fibrations are those maps with the right lifting property with respect to mixed acyclic cofibrations. We use the pushout product to define the generating acyclic cofibrations here; given $f: A \rightarrow B$ and $g: X \rightarrow Y$ then $f \square g$ is the map $A \wedge Y \cup_{A \wedge X} B \wedge X \rightarrow B \wedge Y$.

Proposition 1.3. The monomorphisms, underlying weak equivalences and mixed fibrations form a cofibrantly generated model structure on the category of pointed G-spaces.

Proof. Let $I_{G}=I^{\prime}$ from the proof of Proposition 1.2. The generating acyclic cofibrations require an extra step. Let $\pi_{H}$ be the projection $\left(G \times{ }_{H} E H\right)_{+} \rightarrow$ $(G / H)_{+}$. Use the mapping cylinder construction, see [HSS, 3.1.7] for example, to factor $\pi_{H}$ as $\left(G \times \times_{H} E H\right)_{+} \stackrel{j_{H}}{\longrightarrow} M \pi_{H} \stackrel{r_{H}}{\longrightarrow}(G / H)_{+}$with $j_{H}$ a monomorphism and $(G / H)_{+}$a simplicial deformation retract of $M \pi_{H}$. Let $J_{G}=J^{\prime} \cup\left\{j_{H} \square i_{k}\right\}$ where $i_{k}$ is as in $I^{\prime}, H$ varies over all subgroups of $G$ and $k>0$. We now check the statements required by [Hov98, 2.1.19]. Since the pushout product preserves monomorphisms, $J_{G} \subseteq I_{G^{-c o f}}$. Thus, $J_{G}$-cof $\subseteq I_{G^{-c o f}}$ and $I_{G}$-inj $\subseteq J_{G}$-inj. Since pushouts of $G$-spaces are determined by the underlying pushout of spaces and such pushouts preserve acyclic cofibrations of spaces, it follows that any map in $J_{G}$-cof is an underlying weak equivalence. Since the $I_{G}$-injective maps are equivariant weak equivalences, they are also underlying weak equivalences.

The last statement which we need to check is that any map which is $J_{G}$-injective and an underlying weak equivalence is $I_{G}$-injective. One can check by adjoint-

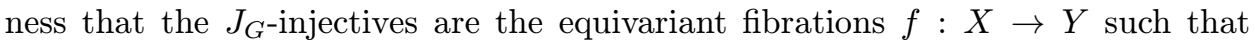
$f^{\pi_{H}}: X^{H} \rightarrow Y^{H} \times_{Y^{h H}} X^{h H}$ is a weak equivalence for each subgroup $H$ in $G$ or equivalently that $f^{j_{H}}$ is an acyclic fibration for each $H$; this uses the fact that $(G / H)_{+}$is a simplicial deformation retract of $M \pi_{H}$, for a similar argument see [HSS, 3.4.12]. If $f$ is also an underlying weak equivalence, then it follows that $f^{h H}$ and hence also $f^{H}$ is a weak equivalence. That is, any acyclic $J_{G \text {-injective is }}$ an equivariant acyclic fibration.

\section{SyMmETRIC SPECTRA}

Given the background material on $G$-spaces we now turn to symmetric sequences $\left(\mathcal{S}_{*}^{\Sigma}\right.$, see [HSS, 2.1]) as one more preparatory step before considering symmetric spectra.

Proposition 2.1. The monomorphisms, level equivalences and level mixed fibrations form a cofibrantly generated model structure on the category of symmetric sequences.

Proof. Let $\bar{G}_{n}: \Sigma_{n}-\mathcal{S}_{*} \rightarrow \mathcal{S}_{*}^{\Sigma}$ be the left adjoint of the evaluation functor which takes a symmetric sequence to the pointed $\Sigma_{n}$-space in level $n$. Note that in the notation of [HSS, 2.1.7], $G_{n}(X)=\bar{G}_{n}\left(X \wedge \Sigma_{n+}\right)$. Let $I^{l}$ be the union of the sets $\bar{G}_{n}\left(I_{\Sigma_{n}}\right)$ and $J^{l}$ be the union of the sets $\bar{G}_{n}\left(J_{\Sigma_{n}}\right)$ for $n \geq 0$. Since $\mathcal{S}_{*}^{\Sigma}$ is just a product category it is easy to check that $I^{l}$ and $J^{l}$ generate a model category on $\mathcal{S}_{*}^{\Sigma}$ with the stated structure. 
Next we lift this model structure to a level $S$-model structure on symmetric spectra. Recall from [HSS, 5.3.6] that an $S$-cofibration is a map of spectra in $(S \otimes M)$-cof where $M$ is the class of monomorphisms in $\mathcal{S}_{*}^{\Sigma}$. A map of symmetric spectra is a level $S$-fibration if it has the right lifting property with respect to every map which is both an $S$-cofibration and a level equivalence.

Proposition 2.2. The $S$-cofibrations, level equivalences and level $S$-fibrations form a cofibrantly generated model structure on the category of symmetric spectra.

Proof. Here we use the lifting lemma for model categories established in [SS00, 2.3]. We consider the adjoint pair of functors between symmetric sequences and symmetric spectra with left adjoint $S \otimes-$ and right adjoint the forgetful functor. Let $S I^{l}=S \otimes I^{l}$ and $S J^{l}=S \otimes J^{l}$. To show that these generate a model structure we need to check that any map in $S J^{l}$-cof is a level equivalence. Let $f: X \rightarrow Y$ be a map in $J_{\Sigma_{n}}$. Then $\left(S \otimes \bar{G}_{n}(X)\right)_{q}=\Sigma_{q} \wedge_{\Sigma_{q-n} \times \Sigma_{n}} S^{q-n} \wedge X$ which is a wedge of copies of $S^{q-n} \wedge X$ since $\Sigma_{q}$ is free over $\Sigma_{q-n} \times \Sigma_{n}$. Since $S^{q-n} \wedge-$ and wedges preserve monomorphisms and underlying weak equivalences we see that $S \otimes \bar{G}_{n}(f)$ is a monomorphism and a level weak equivalence. Since pushouts, colimits and retracts are constructed levelwise, they also preserve maps which are monomorphisms and level weak equivalences.

Note that by tracing through the definitions one can show that the $S I^{l}$-injective maps, or the level $S$-fibrations which are level equivalences, are level equivariant acyclic fibrations as in Proposition 1.2. Also note that since $S I^{l} \subseteq S \otimes M \subseteq S I^{l}$-cof, by [HSS, 5.3.4] we have $S I^{l}$-cof $=(S \otimes M)$-cof.

Since the stable cofibrations in [HSS] are generated by maps of the form $S \otimes$ $G_{n}\left(\partial \Delta[k]_{+} \rightarrow \Delta[k]_{+}\right)$, the following holds.

Lemma 2.3. Any stable cofibration in the sense of [HSS] is an S-cofibration.

We are now finally ready to consider the stable $S$-model structure on symmetric spectra. As in [HSS, 5.3.6], call a map an $S$-fibration if it has the right lifting property with respect to every map which is both an $S$-cofibration and a stable equivalence.

Theorem 2.4. The $S$-cofibrations, stable equivalences and $S$-fibrations form a cofibrantly generated model structure on the category of symmetric spectra.

Proof. We use the criteria for a cofibrantly generated model category from [Hov98, 2.1.19]. Let $S I=S I^{l}$ and let $S J=S J^{l} \cup K$ where $K$ is the set defined in [HSS, 3.4.9] which is built from the stably cofibrant replacements of the maps $F_{n+1} S^{1} \rightarrow F_{n} S^{0}$. First note that each map in $S J$ is an $S$-cofibration. It is easy to see that the maps in $S J^{l}$ are in $S \otimes M$; for the maps in $K$ note that they are stable cofibrations and hence also $S$-cofibrations by Lemma 2.3. Thus $S J$-cof $\subseteq S I$-cof and $S I$-inj $\subseteq S J$-inj. Next note that each map in $S J$ is a monomorphism and a stable equivalence. In the proof of [HSS, 5.4.1] it is shown that such maps are preserved under pushouts, colimits and retracts. Thus the maps in $S J$-cof are all stable equivalences. Because of the level $S$-model structure, we also know that the $S I$-injectives are the level acyclic $S J^{l}$-injectives, or the level equivariant acyclic fibrations. So any $S I$-injective is a stable equivalence.

Finally we must show that the maps which are $S J$-injectives and stable equivalences are $S I$-injective. First note that each of the standard generating acyclic stable cofibrations defined in [HSS, 3.4.9] is in $S J$. Thus any $S J$-injective map is a 
stable fibration. So the stably acyclic $S J$-injective maps are stably acyclic fibrations in the usual model category and hence are level acyclic fibrations. So the stably acyclic $S J$-injective maps are level acyclic $S J$-injective maps. Since $S J^{l}$ is a subset of $S J$, any $S J$-injective map is $S J^{l}$-injective. So any level acyclic $S J$-injective map is a level acyclic $S J^{l}$-injective map. But these are just the $S I$-injectives, so we are done.

Proposition 2.5. The stable $S$-model structure is a monoidal model category which satisfies the monoid axiom.

Proof. The monoidal model structure follows, as noted after [HSS, 5.3.8], by [HSS, 5.3.7(2) and (5)] since any $S$-cofibration is also a level cofibration (monomorphism). The monoid axiom also follows from [HSS, 5.3.7(5)] since any $S$-module is level cofibrant and stably acyclic level cofibrations are preserved under pushouts and colimits by the proof of [HSS, 5.4.1].

It follows from [SS00, 4.1] that the analogous model structure can be formed for $R$-modules where $R$ is any associative symmetric ring spectrum. An $R$-cofibration is a map of $R$-modules in $(R \otimes M)$-cof where $M$ is the class of monomorphisms in $\mathcal{S}_{*}^{\Sigma}$. A map of $R$-modules is a (level) $R$-fibration if it is a (level) $S$-fibration as a map of underlying $S$-modules. For $R$ a commutative symmetric ring spectrum, by [SSO0, 4.1] one can also define a model structure on the category of associative $R$-algebras with weak equivalences and fibrations determined on the underlying category of $R$-modules.

\section{Theorem 2.6.}

(1) The $R$-cofibrations, stable equivalences and $R$-fibrations form a cofibrantly generated model structure on the category of $R$-modules.

(2) For $R$ a commutative symmetric ring spectrum, the $R$-model structure is a monoidal model category which satisfies the monoid axiom.

(3) For $R$ a commutative symmetric ring spectrum, the category of associative $R$-algebras is a cofibrantly generated model category. Every $R$-cofibration of associative $R$-algebras whose source is $R$-cofibrant as an $R$-module is also an $R$-cofibration of $R$-modules. In particular, every $R$-cofibrant associative $R$-algebra is also $R$-cofibrant as an $R$-module.

This theorem shows that the $R$-model structure has the same properties as the standard stable model structure on $R$-modules. Since every $R$-cofibration is a level cofibration and every $R$-fibration is a stable fibration and hence a level fibration, [HSS, 5.5.3] we also have the following.

Proposition 2.7. The $R$-model category of $R$-modules is proper.

Also, the $R$-model structure and the usual stable model structure on $R$-modules are Quillen equivalent.

Proposition 2.8. The identity functor from the stable model structure to the $R$ model structure on $R$-modules is the left adjoint of a Quillen equivalence.

Proof. The analogue of Lemma 2.3 with $S$ replaced by $R$ shows that this left adjoint preserves cofibrations. Since the weak equivalences agree in both structures the Quillen equivalence follows.

Remark 2.9. Analogues of the model structures here could be carried out for orthogonal spectra as well. Because orthogonal spectra use topological spaces, 
instead of mimicking the monomorphisms of simplicial sets, one would use analogues of the generators. The $S$-cofibrations would be generated by $\left\{S \otimes \bar{G}_{n}\left(S_{+}^{k-1} \wedge\right.\right.$ $\left.\left.\left(O_{n} / H\right)_{+}\right) \rightarrow S \otimes \bar{G}_{n}\left(D_{+}^{k} \wedge\left(O_{n} / H\right)_{+}\right)\right\}$. Here one can either let $H$ vary through all subgroups of $O_{n}$ or only vary through the subgroups of $\Sigma_{n}$.

\section{Commutative $R$-Algebras}

As with the standard stable model category, before building a model category on the commutative ring spectra one must consider a "positive" variation. We say a map is a positive $R$-cofibration if it is an $R$-cofibration and it is an isomorphism in level zero. The positive $R$-fibrations are then defined as the maps with the right lifting property with respect to any stably acyclic positive $R$-cofibrations.

Proposition 3.1. The positive $R$-cofibrations, stable equivalences and positive $R$ fibrations form a cofibrantly generated, proper model structure on the category of $R$-modules. If $R$ is a commutative ring spectrum then this is a monoidal model category which satisfies the monoid axiom.

Proof. Define $R^{+} I=R \otimes I^{l+}, R^{+} J^{l}=R \otimes J^{l+}, K_{R}^{+}$and $R^{+} J=R^{+} J^{l} \cup K_{R}^{+}$as the subsets of maps with $n>0$ in the sets defined above. $R^{+} I$ and $R^{+} J$ form the generating (acyclic) cofibrations for the positive $R$-model structure. The arguments here follow just as in the last section by referring to the positive stable model category as developed in [MMSS, 14]. In the intermediary level model category one uses positive level equivalences, i.e. maps which are weak equivalences in all positive levels. Notice that any $R$-cofibration which is an isomorphism in level zero can be built using only the maps in $R^{+} I$.

Theorem 3.2. For $R$ any commutative symmetric ring spectrum there is a cofibrantly generated model structure on the category of commutative $R$-algebras with weak equivalences and fibrations the maps which are underlying stable equivalences and underlying positive $R$-fibrations of $R$-modules. The cofibrations in this model category are referred to as the $R$-alg-cofibrations.

To establish this model category we use the lifting property from [SS00, 2.3] applied to the adjoint pair of functors with right adjoint the forgetful functor from commutative $R$-algebras to $R$-modules. We denote the left adjoint by $\mathbb{P}_{R} ; \mathbb{P}_{R}(M)=$ $\vee_{i \geq 0} M^{(i)} / \Sigma_{i}$ where $M^{(i)}=M \wedge_{R} M \wedge_{R} \cdots \wedge_{R} M$, the $i$ th smash power of $M$ over $R$, with $M^{(0)}=R$. We need to verify that the maps in $\mathbb{P}_{R}\left(R^{+} J\right)$-cof are stable equivalences. We only discuss the case where $R=S$; the general case follows from [DS95, 3.10] since commutative $R$-algebras are just the commutative $S$-algebras under $R$.

We first show that the maps in $\mathbb{P}_{S}\left(S^{+} J\right)$ are stable equivalences by showing that in the source and target the orbit constructions can be replaced by homotopy orbits without changing the homotopy type.

Proposition 3.3. If $X$ is positive $S$-cofibrant, $n>0$ and $\Sigma$ is a subgroup of $\Sigma_{n}$, then for any symmetric spectrum $Y$ the map

$$
\left(E \Sigma_{+} \wedge_{\Sigma} X^{(n)}\right) \wedge Y \rightarrow\left(X^{(n)} / \Sigma\right) \wedge Y
$$

is a stable equivalence.

Proof. First note that if $X=S \otimes \bar{G}_{i}\left(K \wedge\left(\Sigma_{i} / H\right)_{+}\right)$with $i>0$ then in level $z$

$$
\left[\left(X^{(n)} / \Sigma\right) \wedge Y\right]_{z}=\Sigma_{z+} \wedge_{\left(H^{(n)} \rtimes \Sigma\right) \times \Sigma_{z-n i}} K^{(n)} \wedge Y_{z-n i} .
$$


Since $\Sigma_{z}$ is free over the subgroup appearing in the subscript, this is just a wedge of copies of $K^{(n)} \wedge Y_{z-n i}$. Similarly, $\left[\left(E \Sigma_{+} \wedge_{\Sigma} X^{(n)}\right) \wedge Y\right]_{z}$ is the same wedge of copies of $E \Sigma_{+} \wedge K^{(n)} \wedge Y_{z-n i}$ and the map is induced by the weak equivalence from $E \Sigma$ to a point. As in the proofs for [Man, 8.2, 8.10] or [MMSS, 15.5] (compare also [EKMM, III.5.1]) the statement holds for general positive $S$-cofibrant $X$ by induction up the cellular filtration.

We next show that pushouts of maps in $\mathbb{P}_{S}\left(S^{+} J\right)$ are stable equivalences and level cofibrations. Since directed colimits of such maps are again stable equivalences by the proof of [HSS, 5.4.1], it follows that all maps in $\mathbb{P}_{S}\left(S^{+} J\right)$-cof are stable equivalences. Theorem 3.2 then follows from Propositions 3.3 and 3.4 by [SS00, 2.3].

Proposition 3.4. If $f: X \rightarrow Y$ is in $S^{+} J$ and $Z$ is a $\mathbb{P} X$-module, then $Z \rightarrow$ $Z \wedge_{\mathbb{P} X} \mathbb{P} Y$ is a stable equivalence and level cofibration.

Proof. This follows just as in the filtration arguments of [Man, 7.5, 8.6] with only slight modifications to the preparatory statements, so we do not repeat the full argument here. The statements in [Man] concern $G$-equivariant spectra. Here we only consider $G=e$, the trivial group. The first modification needs to be made to [Man, 8.2]. The notation $S^{-x}$ is used there instead of $S \otimes \bar{G}_{x}\left(S^{0} \wedge \Sigma_{x+}\right)$. All of the parts of this proposition hold when $S^{-i}$ is replaced by $S^{-i} / H=S \otimes \bar{G}_{i}\left(S^{0} \wedge\right.$ $\left.\left(\Sigma_{i} / H\right)_{+}\right)$where $H$ is any subgroup of $\Sigma_{i}$. Similarly, instead of $\left(S^{-x}\right)^{(n)} \wedge X$ one can consider $\left[S \otimes \bar{G}_{i}\left(K \wedge\left(\Sigma_{i} / H\right)_{+}\right)\right]^{(n)}$; here $X$ is replaced by $K^{(n)}$. This generalization is necessary because these spectra appear as the source and target of the generating (acyclic) cofibrations for the positive $S$-model structure.

A similar modification needs to be made to [Man, 8.8] with $S^{-x}$ replaced by $S^{-x} / H$. Then the filtration $F_{j}^{i} f$ is

$$
\left(S^{-n}\right)^{(i)} \wedge_{H^{(i)} \rtimes \Sigma_{i}} Y_{j}^{i}=S \otimes \bar{G}_{n i}\left(Y_{j}^{i} \wedge\left(\Sigma_{n i} /\left(H^{(i)} \rtimes \Sigma_{i}\right)\right)_{+}\right) .
$$

The last modification is that [Man, 8.10] should be replaced by Proposition 3.3 above so that it applies to all positive $S$-cofibrant spectra.

Finally, the first statement of [Man, 8.6] holds for positive $S$-cofibrations by using the modified versions of [Man, 8.2, 8.8] discussed above. (The statements of [Man, 8.7, 8.9] hold without any modifications.) The second statement of [Man, 8.6] holds for stably acyclic positive $S$-cofibrations if we use Proposition 3.3 instead of [Man, 8.10] and note that the generating acyclic positive $S$-cofibrations are maps between positive $S$-cofibrant objects. The proposition follows.

This $R$-model structure on commutative $R$-algebras is Quillen equivalent to the usual one.

Proposition 3.5. The identity functor from the stable model structure [Man, 7.6] (see also [MMSS, 15.2]) to the $R$-model structure on commutative $R$-algebras is the left adjoint of a Quillen equivalence.

Proof. Since the positive stable generating cofibrations from [MMSS, 15.2] are a subset of the $R$-model generating cofibrations, the positive stable cofibrations are $R$-alg-cofibrations. Since the weak equivalences agree in both structures, the Quillen equivalence follows. 


\section{Properties of $R$-Cofibrations}

Throughout this section let $R$ be a commutative symmetric ring spectrum. First we consider the crucial property that the maps underlying $R$-alg-cofibrations are $R$-cofibrations. This is the main reason for developing this whole new system of model structures.

Proposition 4.1. If $f$ is an $R$-alg-cofibration of commutative $R$-algebras then the underlying map of $f$ on $R$-modules is a positive $R$-cofibration (and hence also an $R$-cofibration).

Proof. First note that $\mathbb{P}_{R}\left(R^{+} I\right)=\mathbb{P}_{R}\left(R \otimes I^{l+}\right)=R \otimes \mathbb{P}\left(I^{l+}\right)$. If we show that the maps in $\mathbb{P}\left(I^{l+}\right)$ are monomorphisms, then it follows that the underlying maps in $\mathbb{P}_{R}\left(R^{+} I\right)$ are positive $R$-cofibrations. Also any $\mathbb{P}_{R}\left(R^{+} I\right)$-cofibration would be a positive $R$-cofibration. The maps in $\mathbb{P}\left(I^{l+}\right)$ are coproducts of maps of the form

$$
G_{n i}\left(\left(\partial \Delta[k]_{+}\right)^{(i)} \wedge\left(\Sigma_{n i} / H^{(i)}\right)_{+}\right) \rightarrow G_{n i}\left(\left(\Delta[k]_{+}\right)^{(i)} \wedge\left(\Sigma_{n i} / H^{(i)}\right)_{+}\right)
$$

with $n>0$. Since each of these pieces is a monomorphism the maps in $\mathbb{P}\left(I^{l+}\right)$ are also monomorphisms. Thus, underlying any $R$-alg-cofibration is a positive $R$ cofibration. The last comment holds because any positive $R$-cofibration is also an $R$-cofibration.

Proposition 4.2. If $f$ is a cofibration in the model structure from [MMSS, 15.2] on commutative $R$-algebras then it is also an $R$-alg-cofibration. Thus, the underlying map of $f$ on $R$-modules is a positive $R$-cofibration (and also an $R$-cofibration). Moreover, the underlying map of $f$ is not necessarily a positive stable cofibration (as defined in [MMSS].)

A related statement appeared in [Sch01, 4.3]; it follows from this statement and Lemma 4.8 .

Proof. The first statement follows since the positive stable cofibrations (from [MMSS, 14.2]) are a subset of the positive $S$-cofibrations. The second statement then follows from Proposition 4.1. For the third statement, one can show that $\mathbb{P}\left(F_{1} S^{1}\right)$ is not positive stably cofibrant as an underlying $S$-module by checking the criterion given in [HSS, 5.2.2]. Specifically, $\left[\left(F_{1} S^{1}\right)^{(2)} / \Sigma_{2}\right]_{2}=\left(S^{1} \wedge S^{1}\right) / \Sigma_{2}$ which is not $\Sigma_{2}$-free.

Since fibrations of commutative $R$-algebras are defined to be those maps which are underlying positive $R$-fibrations, we can conclude from Proposition 4.1 that cofibrations and fibrations of commutative $R$-algebras are underlying positive $R$ cofibrations and positive $R$-fibrations of $R$-modules. Unfortunately this doesn't translate as easily to cofibrant objects because the initial commutative $R$-algebra is $R$ whereas the initial $R$-module is trivial. We do have the following though.

Corollary 4.3. If $A$ is cofibrant as an $R$-algebra, then $A$ is $R$-cofibrant as an $R$ module. If $A$ is fibrant as an $R$-algebra, then $A$ is positive $R$-fibrant as an $R$-module.

Proof. Since $A$ is $R$-alg-cofibrant, the map $i: R \rightarrow A$ is an $R$-alg-cofibration. By Proposition 4.1, $i$ is also a positive $R$-cofibration and an $R$-cofibration. Since $R$ is $R$-cofibrant it follows that $A$ is also. The second statement follows from the fact that fibrations of commutative $R$-algebras are defined to be those maps which are underlying positive $R$-fibrations. 
Note that $R$ is not positive $R$-cofibrant, so we cannot conclude that $A$ is positive $R$-cofibrant. Also, not all positive $R$-fibrations are $R$-fibrations (since $R^{+} J$ is a proper subset of $R J$ ), so $A$ is not necessarily $R$-fibrant. So even if $A$ is cofibrant and fibrant as an $R$-algebra, $A$ is not necessarily both cofibrant and fibrant in any one model category on $R$-modules. Because of the next proposition though, this turns out to be enough to still produce morphism spectra with the right homotopy type.

The necessary properties on the morphism spectra follow from properties of the monoidal product. We consider the closed symmetric monoidal structure on $R$-modules as constructed in [HSS, 2.2.4, 5.4.2]; see also Theorem 2.6 (2) and Proposition 3.1. Here we show that the action of the usual $R$-model structure on the positive $R$-model structure is compatible with this monoidal structure; see [Hov98, 4.2.18] for a general definition which extends [Qui67, SM7].

Proposition 4.4. If $i$ is a positive $R$-cofibration and $j$ is an $R$-cofibration then the pushout product $i \square j$ is a positive $R$-cofibration which is acyclic if either $i$ or $j$ is. That is, the positive R-model structure is a Quillen module over the (non-negative) $R$-model structure on $R$-modules.

Proof. Since any positive $R$-cofibration is also an $R$-cofibration and the $R$-model structure is a monoidal model category by Theorem $2.6(2)$ we only need to check that the pushout product of a positive $R$-cofibration with an $R$-cofibration is an isomorphism in level zero (and hence a positive $R$-cofibration instead of just an $R$ cofibration.) This is easy to check on the generators since they are maps between free $R$-modules.

Using adjunction and lifting properties this compatibility also extends to the morphism spectra by [Hov98, 4.2.2]. Given maps of $R$-modules $i: A \rightarrow B$ and $p: X \rightarrow Y$ then as in [HSS, 5.3] define $\operatorname{Hom}_{R}(i, p)$ to be the map $\operatorname{Hom}_{R}(B, X) \rightarrow$ $\operatorname{Hom}_{R}(B, Y) \times_{\operatorname{Hom}_{R}(A, Y)} \operatorname{Hom}_{R}(A, X)$.

\section{Corollary 4.5.}

(1) If $i$ is a positive $R$-cofibration and $p$ is a positive $R$-fibration, then $\operatorname{Hom}_{R \square}(i, p)$ is an $R$-fibration which is acyclic if either $i$ or $p$ is.

(2) If $i$ is an $R$-cofibration and $p$ is a positive $R$-fibration, then $\operatorname{Hom}_{R \square}(i, p)$ is a positive $R$-fibration which is acyclic if either $i$ or $p$ is.

Specifically, this implies the following statement. Since a cofibrant and fibrant commutative $R$-algebra is $R$-cofibrant and positive $R$-fibrant by Corollary 4.3 , this is the homotopy invariance statement needed in [GS].

Corollary 4.6. If $X$ is $R$-cofibrant and $Y$ is positive $R$-fibrant, then $\operatorname{Hom}_{R}(X, Y)$ is weakly equivalent to the derived morphism spectrum. In particular, for $R=S$ it has the same homotopy type as the standard derived morphism spectrum on the homotopy category of spectra.

Proof. Let $Y \rightarrow Y^{f}$ be the $R$-fibrant replacement of $Y$. Since $R$-fibrant objects are also positive $R$-fibrant, $\operatorname{Hom}_{R}(X, Y) \rightarrow \operatorname{Hom}_{R}\left(X, Y^{f}\right)$ is a stable equivalence by Corollary 4.5 (2) and Ken Brown's Lemma [Hov98, 1.1.12]. Since $X$ is $R$-cofibrant and $Y^{f}$ is $R$-fibrant, this means $\operatorname{Hom}_{R}(X, Y)$ has the same homotopy type as the derived morphism spectrum induced by the monoidal $R$-model structure on $R$ modules; see [Hov98, 4.3.2]. Note, by Corollary $4.5(2) \operatorname{Hom}_{R}(X, Y)$ is positive 
$R$-fibrant. So although $\operatorname{Hom}_{R}(X, Y)$ is the right homotopy type, its zeroth level may not be.

To see that this derived morphism spectrum is independent of the model for $R$-modules, note that Proposition 2.8 can be generalized to show that the $R$-model structure is monoidally equivalent [Hov98, 4.2.16] to the usual stable structure on symmetric spectra since the identity functor is monoidal. Then [Hov98, 4.3.3] and the various comparisons of symmetric monoidal categories of spectra in [MMSS, Sch01, MM02] provide the further comparisons. Since [MMSS, 0.3] shows that the derived closed symmetric structure on symmetric spectra agrees with the standard structure on the homotopy category of spectra, the second statement follows.

We end this section by stating some of the useful properties of the $R$-model structure on commutative $R$-algebras.

Proposition 4.7. The category of commutative $R$-algebras is proper. It follows that if $f: A \rightarrow B$ is a weak equivalence of commutative $R$-algebras, then restriction and extension of scalars induces a Quillen equivalence between the categories of commutative A-algebras and commutative B-algebras.

We use the following lemma to prove left properness. It is also useful in comparing $R$-alg-cofibrations for various $R$. The statement follows by the definition of the $R$-model category on commutative $R$-algebras as the subcategory under $R$ in commutative $S$-algebras.

Lemma 4.8. Any R-algebra map which is an $S$-alg-cofibration on the underlying $S$-algebras is an $R$-alg-cofibration.

Proof of Proposition 4.7. Since a fibration of $R$-algebras is an underlying positive $R$-fibration, right properness follows from a generalization of [HSS, 5.5.3] to positive level fibrations. For left properness consider an $R$-alg-cofibration $i: A \rightarrow B$ and a stable equivalence $j: A \rightarrow C$. Note that since $i$ is an $R$-alg-cofibration and a map of $A$-algebras it is an $A$-alg cofibration by Lemma 4.8 . Thus $B$ is an $A$-cofibrant algebra and hence an $A$-cofibrant $A$-module by Corollary 4.3. We want to show that the pushout of $j$ over $i, B=B \wedge_{A} A \rightarrow B \wedge_{A} C$, is a stable equivalence. This follows from the $A$-module analogue of [HSS, 5.3.10] which shows that $B \wedge_{A}-$ preserves stable equivalences for $B$ an $A$-cofibrant $A$-module.

Restriction of scalars from commutative $B$-algebras to commutative $A$-algebras preserves weak equivalences and fibrations since these are all defined on the underlying $S$-algebras. For $C$ any cofibrant $A$-algebra, $C \rightarrow B \wedge{ }_{A} C$ is a weak equivalence by left properness. Thus if $D$ is any $B$-algebra then an $A$-algebra map $C \rightarrow D$ is a weak equivalence if and only if the adjoint $B$-algebra map $B \wedge_{A} C \rightarrow D$ is a weak equivalence. The Quillen equivalence follows by definition [Qui67, I.4 Theorem 3]; see also [DS95, 9.7].

Since the category of commutative $A$-algebras is a symmetric monoidal category, one can consider the compatibility of the monoidal structure with the cofibrations. Since the monoidal product is the coproduct here though, this is trivial. In particular, the pushout product of any two maps is the identity on the monoidal product of the targets. So any model structure on commutative $A$-algebras is a monoidal model category since the pushout product of any two maps is an isomorphism. One useful piece of this structure is the usual property that the coproduct of cofibrant objects is cofibrant. 
Proposition 4.9. If $B$ and $C$ are cofibrant commutative $A$-algebras then $B \wedge_{A} C$ is a cofibrant A-algebra as well.

\section{REFERENCES}

[Bas99] M. Basterra, André-Quillen cohomology of commutative S-algebras, J. Pure Appl. Algebra 144 (1999), no. 2, 111-143.

[Bor94] F. Borceux. Handbook of categorical algebra II, Categories and structures, Cambridge University Press, 1994.

[DHK] W. G. Dwyer, P. S. Hirschhorn, and D. M. Kan, Model categories and general abstract homotopy theory, in preparation.

[DS95] W. G. Dwyer and J. Spalinski, Homotopy theories and model categories, Handbook of algebraic topology (Amsterdam), North-Holland, Amsterdam, 1995, pp. 73-126.

[EKMM] A. D. Elmendorf, I. Kriz, M. A. Mandell, and J. P. May, Rings, modules, and algebras in stable homotopy theory. With an appendix by $M$. Cole, Mathematical Surveys and Monographs, vol. 47, American Mathematical Society, Providence, RI, 1997, xii+249 pp.

[GS] J. P. C. Greenlees and B. Shipley, Rational torus-equivariant stable homotopy III: the Quillen equivalence, in preparation.

[Hov98] Mark Hovey, Model categories, Mathematical Surveys and Monographs, vol. 63, American Mathematical Society, Providence, RI, 1998.

[HSS] M. Hovey, B. Shipley, and J. Smith, Symmetric spectra, J. Amer. Math. Soc. 13 (2000), 149-208.

[MM02] M. A. Mandell and J. P. May, Equivariant Orthogonal Spectra and S-Modules, Mem. Amer. Math. Soc. 159 (2002), no. 755, x+108 pp.

[MMSS] M. A. Mandell, J. P. May, S. Schwede and B. Shipley, Model categories of diagram spectra, Proc. London Math. Soc. 82 (2001), 441-512.

[Man] M. A. Mandell, Equivariant symmetric spectra, preprint 2002, submitted to these same Northwestern conference proceedings.

[Qui67] Daniel G. Quillen, Homotopical algebra, Lecture Notes in Mathematics, vol. 43, Springer-Verlag, 1967.

[Sch01] Stefan Schwede, S-modules and symmetric spectra, Math. Ann. 319 (2001), 517-532.

[SS00] S. Schwede and B. Shipley, Algebras and modules in monoidal model categories, Proc. London Math. Soc. 80 (2000), 491-511.

Department of Mathematics, Purdue University, West Lafayette, IN

E-mail address: bshipley@math.purdue.edu 